Horizons philosophiques

\title{
La sémiotique, les objets singuliers et la complexité
}

\section{Gilles Thérien}

Volume 1, numéro 2, printemps 1991

Sémiotiques 2 : théories et champs d'application

URI : https://id.erudit.org/iderudit/800871ar

DOI : https://doi.org/10.7202/800871ar

Aller au sommaire du numéro

\section{Éditeur(s)}

Collège Édouard-Montpetit

\section{ISSN}

1181-9227 (imprimé)

1920-2954 (numérique)

Découvrir la revue

Citer cet article

Thérien, G. (1991). La sémiotique, les objets singuliers et la complexité.

Horizons philosophiques, 1(2), 33-49. https://doi.org/10.7202/800871ar d'utilisation que vous pouvez consulter en ligne.

https://apropos.erudit.org/fr/usagers/politique-dutilisation/ 


\section{La sémiotique, les objets singuliers et la complexité}

Depuis un quart de siècle environ, la sémiotique qui se développe en Europe, en France en particulier, emprunte ses principales notions à la linguistique saussurienne : le signifiant et le signifié, la langue et la parole, le syntagme, la diachronie, le paradigme, la valeur, l'arbitraire du signe, etc. Aujourd'hui, personne ne sent vraiment le besoin de savoir si tel ou tel usage est bien conforme à la pensée de Saussure. L'œuvre de Greimas, les travaux de sémiolinguistique ont conquis leur indépendance. Nous acceptons volontiers qu'avec le temps et, en face de problèmes cernés de plus en plus finement, il soit devenu futile de maintenir une pseudo orthodoxie saussurienne qui ne servirait qu'à ralentir la recherche. La question sémiotique se déporte du côté des objets. Sont-ils mieux compris, mieux décrits, mieux expliqués à travers les diverses méthodes d'inspiration saussurienne? On le sait, certains en doutent. Aussi ne s'étonnera-t-on pas de voir une nouvelle attention portée à l'autre père fondateur de la sémiotique, Charles Sanders Peirce.

II faut reconnaître l'importance grandissante des études peirciennes mais, avec elles, une tendance normale et inévitable à dégager une orthodoxie peircienne, orthodoxie d'autant plus difficile à définir selon que Peirce fait 
l'objet d'une exégèse philosophique ou d'une lecture sémiotique. On ne peut certes pas réduire la pensée de Peirce à la semiosis ou plutôt à l'emploi fétichiste de ce terme que l'on ne prend pas toujours le soin de comprendre ou de définir, en montrant en quoi il garde la trace peircienne ou en quoi il s'écarte de son modèle. Le progrès de la sémiotique devrait nous permettre - ce serait d'ailleurs conforme à notre perspective méthodologique 1 - de revoir Peirce, de le relire avec une lecture contemporaine, et de l'exploiter en fonction des problématiques que nous définissons et non en fonction d'un champ philosophique défini au siècle dernier. Nous pouvons minimalement nous autoriser de la lecture de Peirce par Charles Morris pour prendre un certain nombre de libertés. C'est dans cet esprit que j'aborde la notion d'objet et son utilisation sémiotique possible sous l'angle d'objet singulier. J'illustrerai mon propos de deux exemples pris chez Barthes.

La pensée peircienne est d'essence philosophique. La sémiotique n'en constitue qu'une partie que Peirce définit avec soin, mais qu'il n'est pas toujours facile de distinguer d'une théorie de la connaissance. Or, ce point, me semble-t-il, est essentiel pour le développement de la sémiotique. Si les sémioticiens ne peuvent pas être ignorants des théories de la connaissance, ces dernières ne sont quand même pas leur principal et ultime objet de recherche. Ils ont la tâche, ardue certes, de distinguer le signe de ce qui n'en est pas, de l'isoler et d'en déterminer les règles de fonctionnement sans avoir à valider une théorie de la connaissance en particulier. Les théories de la connaissance sont les contextes épistémologiques et les contextes

1. Celle d'une sémiotique de la lecture [Thérien 1990]. 
d'usage du signe, et doivent être traités au niveau où ils se posent, dans le cadre d'une théorie philosophique et de la psychologie.

II existe un autre problème qui risque de fausser notre point de vue. La sémiotique peircienne est pensée dans un ensemble plus vaste axé sur le développement des sciences, de la pensée scientifique et de la poursuite de la vérité scientifique. Les rapports qu'entretient la semiosis avec les divers modes de raisonnement sont influencés par cette position historique et, ici encore, il nous semble particulièrement utile de pouvoir prendre nos distances. La sémiotique à laquelle chacun travaille actuellement n'est pas de toute évidence scientifique et ne peut pas utiliser les divers modes de raisonnement théoriquement mis à sa disposition. De plus, le choix des corpus d'expérimentation, en particulier dans le domaine des sciences humaines, introduit un facteur aléatoire qui rend nécessaire une interrogation sur le statut de nos objets. D'où la présente réflexion. Comment concilier l'analyse d'un objet quelconque et la notion d'objet telle qu'on la trouve dans la sémiotique peircienne?

L'objet au sens peircien du terme se comprend de diverses façons. II apparaît comme l'objet référentiel dont on est en train de parler; il peut tout aussi bien être compris comme l'occurrence concrète d'un objet. II peut être pensé comme faisant partie de la semiosis ou comme étant à l'extérieur. Peirce parle d'objet immédiat et d'objet dynamique. Chacun de ces objets peut être examiné selon la priméité, la secondéité ou la tiercéité. Nous pouvons chercher à établir ce que Peirce voulait exactement dire par objet, - il emploie d'ailleurs parfois l'expression "corrélat» - ou nous pouvons, pour les besoins de la construction sémiotique, utiliser une notion d'objet qui semble pouvoir mieux 
répondre à nos besoins. C'est cette dernière voie que nous suivrons en étant bien conscient que notre point de vue est forcément une relecture de Peirce, lecture enrichie de tout ce qui nous sépare de lui.

L'objet de la semiosis n'est pas l'objet réel existant en dehors de toute considération cognitive. II est l'objet de la semiosis en ce sens qu'il est le résultat d'une construction entre le fondement de l'interprétance et l'interprétance ellemême. Rattacher l'objet à l'objet réel, c'est rabattre la théorie peircienne sur des théories de la connaissance de type réaliste, où l'objet est premier et prend à sa charge son pouvoir d'autoreprésentation face à une interprétance qui est placée en position passive par rapport au phénomène de la connaissance. Nous l'avons dit plus haut, il ne s'agit pas pour nous de revenir à une théorie de la connaissance, mais d'exploiter un système sémiotique qui permet de comprendre comment les signes se forment, interagissent, produisent de la connaissance et de l'action. Toujours dans le même sens, il se peut, il arrive même fréquemment dans nos domaines «mous", que l'objet sémiotique n'ait pas d'équivalent référentiel externe. Nous reviendrons sur cette question dans nos exemples.

L'objet interne à la sémiosis, nous le pensons comme l'objet immédiat. Mais, puisque, par définition, la semiosis est un processus complexe qui permet l'acquisition de nouvelles connaissances par la construction de nouveaux signes ou systèmes de signes, l'objet immédiat n'est que le versant interne de la semiosis qui pose, dynamiquement, comme un fait brut, incontournable, l'existence d'un autre objet, l'objet dynamique, celui vers lequel tend la semiosis, objet qui n'est jamais atteint puisque toujours déporté par une semiosis qui, dans son approche, le complexifie et, ce faisant, empêche la réduction cognitive qui ferait de l'objet dynamique l'objet réel. Nous comprenons la tension entre l'objet immédiat et l'objet dynamique comme la référence, c'est-à-dire l'établissement d'une corrélation entre un objet 
obtenu par l'activité sémiotique et l'exploration d'un objet impossible à cerner parfaitement.

L'activité sémiotique, dans la perspective de Peirce, est partie prenante de l'établissement du savoir. Or, si le but poursuivi par la science est l'établissement de lois par lesquelles un savoir saura rendre compte de tous ses objets, il nous apparaît important de soulever la question de la difficulté d'obtenir ce résultat qui ressemble plus à celui que l'on peut escompter dans les sciences dures que dans le domaine plus mou des sciences humaines. En effet, comment rendre compte d'un objet textuel? On connaît, depuis trente ans, toutes les tentatives, inspirées d'une visée néopositiviste, de faire entrer l'objet textuel dans des cadres scientifiques. Or, il nous semble que certains objets se réduisent mal à l'abstraction nécessaire de leur singularité pour pouvoir devenir simplement des lieux d'application d'une loi plus générale. La déduction ne produit pas la singularité. Elle applique, pour reprendre les expressions de Peirce, un type à une occurrence, au token. II nous faut donc poser la question de l'analyse de la singularité par l'activité sémiotique et proposer une autre façon de procéder.

En prenant l'exemple de l'objet littéraire, je reporte la singularité de l'objet strictement dans la tension continue entre l'objet immédiat et l'objet dynamique, en utilisant des savoirs qui, eux, peuvent être formulés selon certaines lois, savoir cognitif, savoir argumentatif, savoir narratologique, mais dont l'application n'a pas pour but de ramener l'objet récalcitrant à des proportions plus modestes, mais, au contraire, de le complexifier de telle façon que l'objet devienne sa propre loi ou, pour reprendre les termes peirciens, que le type devienne équivalent au token. Cela veut dire que, pour le sémioticien littéraire par exemple, la tâche importante relève de l'analyse de la singularité, de la collection de singularités, de la formation de séries de singularités dont nous cherchons à rendre compte en construisant sans 
cesse un objet immédiat qui fuit en direction du toujours inatteignable et toujours plus complexe objet dynamique. L'aspect scientifique de la méthode devra plutôt se retrouver dans la constitution même de la singularité que dans sa réduction à des caractéristiques plus générales. II serait ainsi pensable de distinguer divers parcours de la discipline sémiotique selon les niveaux de tiercéité où elle s'applique, et nous dirons ici que, dans le cas de l'objet littéraire singulier, la tiercéité s'applique à la secondéité, les savoirs sont au service du singulier et non le contraire.

J'ai choisi deux exemples dans Barthes. D'abord les Fragments d'un discours amoureux [1977] puis La Chambre claire [1980]. Ce choix tient à ce que les deux objets peuvent servir à la fois d'exemplum de la théorie sémiotique et de champ d'application. En effet, compte tenu de ce que nous avons dit plus haut sur la nature de l'objet, on peut définir le signe de façon globale comme la relation constituante entre l'objet du monde et sa représentation dans le sujet. Le signe est la présence paradoxale d'un objet absent. Le signe n'existe jamais seul, il n'est jamais transparent, il n'est jamais univoque à moins d'avoir été contraint "conventionnellement» à l'être, par exemple en mathématique. Les signes n'ont pas un usage unique, prédéterminé. Ils sont économiques au sens où ils peuvent servir dans plusieurs systèmes très différents les uns des autres. Le signe est toujours un signe en contexte. II suppose toujours l'existence d'au moins un deuxième signe. II forme système, si petit soit-il. La fonction du système de signes est de représenter (figurer) tant l'objet absent antérieur, l'objet réel, le livre ici, que l'objet absent postérieur, sa signification, son sens, sa valeur esthétique.

Fragments d'un discours amoureux est un texte qui porte sur l'absence, nous le verrons, une double absence. 
II est le signe de quelque chose qui n'est pas là, à la poursuite duquel le texte se construit mais qui demeure toujours inatteignable. Double absence parce que ce texte porte deux marques concrètes de cette absence : le fragment comme mode d'écriture qui renvoie à une totalité absente et l'image-fragment qui occupe la couverture du livre $^{2}$. Le lecteur, seul dépositaire de l'activité sémiotique, doit donner sens à ces divers éléments.

L'absent, c'est le thème du deuxième fragment. Par le premier, "Je m'abîme, je succombe...", Barthes a déjà établi qu'à l'origine se trouve cet abîme fait à la fois de bonheur ou de blessure dont les caractéristiques finissent par être les mêmes pour l'amoureux. L'absent, c'est l'autre, c'est le "tu", et, subtilement, Barthes propose que, le «je" étant toujours présent, c'est le "tu» qui peut s'absenter. Les deux positions ne peuvent permuter. II faut alors comprendre que les pronoms ne sont pas des cases absolument vides où, comme dans un dialogue, chacun dit tour à tour "je", mais ils sont des positions fixes où, par définition, le «je» est l'amoureux, le principe actif, la permanence, la fidélité. En somme, c'est ce que Barthes appellera une "structure habitable». Cette structure est habitée normalement par la femme sédentaire, Pénélope, qui tisse pendant qu'Ulysse, nomade, navigue. Le "je» amoureux est une structure féminine et l'homme qui parle l'absence de l'autre, se féminise. Il est intéressant de noter les glissements que Barthes fait subir à la théorie de Benveniste pour amarrer dans les sexes les shifters pronominaux. De plus, grâce au personnage de Werther convoqué à titre d'exemple, il peut utiliser la troisième personne tout

2. Sur la couverture, un fragment qui représente deux personnages fragmentés qui se tiennent par le bras, celui de gauche porte une robe et sa manche est couverte de dentelles, celui de droite est plus réduit encore ne montrant que son bras articulé sur celui de l'autre et une partie de son pourpoint. II s'agit en apparence d'un couple où la femme est à gauche et l'homme à droite. 
en laissant se profiler la silhouette de Charlotte reformant un couple qui n'en est déjà plus un. La troisième personne, celle de l'absence, ce sera Werther, la plupart du temps, l'autre-à-reconstituer, mais ce sera aussi « $X$ » qui n'est jamais nommé autrement que par cette absence de nom, "X» étant aussi l'inconnu[e] en algèbre. Nous nous retrouvons avec deux figures pronominales de l'absent, le "tu", "l'homme nomade", et le véritable absent de la scène du discours, $\mathrm{X}$, dont Werther serait une figuration archétypale au plan romanesque. Pourtant l'arrangement discursif de ce fragment pointe dans une autre direction : ne faudrait-il pas croire que Werther, amoureux, est une figure du «je», comme le "tu» pourrait être Charlotte. II n'y a que le lecteur pressé qui retiendra cette hypothèse dont l'effet serait de détruire la simulation du discours amoureux. Non seulement les positions de langage seraient-elles prises une fois pour toutes, mais, encore, on connaîtrait à l'avance l'issue du dialogue.

L'absent du «je», c'est le «tu», et l'absent du «tu», c'est le «je». Au premier, il manque l'homme; au second, la féminité. Alors entrent en scène les deux autres figures de l'absent, la mère et l'androgyne. Entre le «je» et le «tu» s'établit une relation horizontale; avec la mère, il s'agit d'une relation verticale. La première perception de l'altérité chez l'enfant se fait par le détachement progressif de la mère, chacun ayant son corps propre. L'épisode de la bobine, du fort-da, est postérieur. C'est la crainte du petit d'homme de rester démuni en cas d'absence de la mère, et notons alors que c'est la mort qui est absence et non le contraire. Être dressé à supporter l'absence, c'est absenter la mort. Le meilleur dressage est celui de l'infidélité, du recours à l'homme en soi. Le drame de l'amoureux, c'est qu'il ne peut pas oublier et qu'il nourrit son infidélité de souvenirs : «Enfant, je n'oubliais pas : journées interminables, journées abandonnées, où la Mère travaillait loin; j'allais, le soir, attendre son retour à l'arrêt de 
l'autobus Ubis, à Sèvres-Babylone; les autobus passaient plusieurs fois de suite, elle n'était dans aucun ${ }^{3}$ ". On notera deux choses à propos de cet extrait : il est cité entre parenthèses et le mot "mère» $y$ apparaît avec une majuscule, ce qui n'est pas le cas lorsqu'il est question de la mère en général, mais qui rejoint la pratique du $F$ majuscule à la Femme qui attend. La Mère est l'absente. Elle est du côté du "tu", du côté de l'homme chasseur, voyageur. Le souvenir de l'enfant doublé de celui de l'adulte est du côté de l'amoureux, du féminin sédentaire. La mère change de rôle sans changer de sexe. C'est le père décédé que la mère remplace dans la journée en travaillant loin. Elle rend la structure inhabitable par une oscillation entre les deux pôles du genre mis en contradiction avec les rôles pronominaux tels que Barthes les a ici définis. La Mère figure l'androgyne. Elle est les deux moitiés, père et mère. Quand elle réintègre la position du «je», il y a, pour elle aussi, un absent, dans sa propre structure horizontale, le mari, le «tu» qui ne reviendra jamais de la guerre, du voyage, de la chasse. En un sens, c'est lui l'éternel absent, lui dont Barthes n'a pu rien vérifier, ni la chaleur du geste, ni la clarté du regard, ni le grain de la voix. Le père est réduit à deux photos non inclusives de la relation père-fils. C'est RB par rb [1975] qui se donne un père. Mais il y a plus. Dans la manipulation de l'absence, telle que décrite par Freud, le moment difficile de la séparation mimée par le "fort» est alterné avec celui, joyeux, du retour. Or, dans le texte de Barthes, le discours amoureux ne se construit que sur le "fort". La bobine ne revient pas. Elle ne revient jamais au point où le livre va se terminer sur une évocation du «non vouloir saisir» 4 , position ascétique qui rappelle le zen, mais qui marque aussi quelle attitude doit avoir le
3. P. 21.
4. P. 275. 
sujet quand le retour, le «da", n'est pas possible. On notera aussi que le fragment sur l'absent se termine par un koan bouddhique dont l'utilisation ne manque pas de surprendre : Barthes identifie au Maître zen l'absence de l'autre qui lui tient la tête sous l'eau jusqu'à ce que le disciple comprenne ce que signifie le besoin de vérité, comme le besoin d'air, jusqu'à ce qu'il comprenne que sa vérité à lui, c'est l'intraitable de l'amour, ce qui ne peut être dit parce que ça ne peut être su. L'autre, c'est le Maître, non la Mère.

Il est difficile de terminer ce bref commentaire du Fragment d'un discours amoureux sans souligner l'emploi d'une métaphore lourde de conséquence pour la poursuite de la recherche de Barthes : "Les deux moitiés de l'androgyne soupirent l'une après l'autre : image de l'embrassement, en tant qu'il fond les deux images en une seule : dans l'absence amoureuse, je suis, tristement, une image décollée, qui sèche, jaunit, se recroqueville ${ }^{5}$ ». II s'agit bien ici de l'image photographique, mais de quoi est-elle décollée, de sa moitié, de son support, de son référent? L'image comme le discours est fragmentée et fragmentaire. Et cela nous conduit au deuxième exemple des Fragments, l'image de couverture qui présente ce qui semble être un couple dont on ne distingue qu'une partie des corps et les bras qui cheminent se tenant l'un l'autre. La référence de ce tableau est étonnante puisqu'elle identifie le tableau comme Tobias et l'Ange 6 et donc ramène le pseudo-couple du fragment de la couverture à un épisode biblique où Tobie se fait accompagner d'un ange en voyage avec un double objectif, régler un problème d'argent pour son père aveugle et se trouver une épouse. Ce qui apparaissait

\section{P. 21.}

6. Couverture de fin : «En couverture : "Tobias et l'Ange", Atelier de Verrochio, The National Gallery, Londres.» 
d'abord comme la partie féminine du couple s'avère être un ange asexué, provisoirement chargé d'un corps; il ne reste que le jeune Tobie parti pour accomplir l'ordre de son père. Cette image, dont la totalité est cachée au lecteur, dont le fragment divertit le sens, vient, comme en supplément, en bout de lecture, reprendre sur le mode du secret, l'inévitable absence de l'autre tout en lui conférant un caractère d'impossibilité par la formation d'un couple qui ne peut manifestement pas en être un et en rendant absente la future femme de Tobie, Sara, redoutable, dont les sept premiers maris sont morts "le premier soir quand ils entraient dans sa chambre ${ }^{7}$. L'objet singulier que sont les Fragments d'un discours amoureux nous renvoie tant par les fragments discursifs que par le fragment pictural à un objet de plus en plus complexe, le sens même du livre, le sens pour un lecteur qui, en multipliant ses lectures, ne peut que rendre ce sens toujours plus complexe et toujours plus inatteignable.

Le deuxième exemple est tiré de La Chambre claire. On aurait pu croire, après l'expérience du discours amoureux, que Barthes suivrait la même voie autour des fragments discursifs et de la présence refusée au lecteur d'une photo de la mère. Ce serait alors redoubler la problématique déjà présente dans les Fragments. Le hasard de la lecture a voulu qu'il se trouve une autre piste tout aussi intéressante et non moins troublante.

Dans La Chambre claire, Barthes cite 26 photographes et reproduit 25 illustrations de 15 des photographes cités. Les deux photographes les plus cités sont Kertész et Mapplethorpe, chacun sept fois. Deux photos de Mapplethorpe sont reproduites dans le livre. Depuis sa mort, Mapplethorpe est devenu, surtout aux USA, un objet de scandale public au moment de rétrospectives de son

7. Livre de Tobie, ch. 7, verset 11. 
œuvre. Or, à travers la lecture de La Chambre claire, il était difficile de tirer une impression de scandale. La première photo est celle de Philip Glass et Robert Wilson. Elle date de 1976. La seconde est intitulée par Barthes «Jeune homme au bras étendu". II s'agit en fait d'un autoportrait de Mapplethorpe, qui date de 1975. Curieusement, dans La Chambre claire, il est présenté comme la partie gauche du corps alors que dans les catalogues de l'œuvre de Mapplethorpe, il s'agit plutôt de la partie droite. II y aurait eu inversion non remarquée ou voulue puisque répétée dans les impressions subséquentes de cette photo. Les fragments discursifs qui accompagnent ces deux photos sont particulièrement intéressants. Dans le cas de la photo de Glass et de Wilson, nous lisons : "Ce que je peux nommer ne peut réellement me poindre. L'impuissance à nommer est un bon symptôme de trouble. Mapplethorpe a photographié Bob Wilson et Phil Glass. Bob Wilson me retient, mais je n'arrive pas à dire pourquoi, c'est-à-dire où: est-ce le regard, la peau, la position des mains, les chaussures de basket? L'effet est sûr, mais il est irrepérable, il ne trouve pas son signe, son nom; il est coupant et atterrit cependant dans une zone vague de moi-même; il est aigu et étouffé, il crie en silence. Bizarre contradiction : c'est un éclair qui flotte ${ }^{8}$ ". Le deuxième fragment est encore plus intéressant : "La présence [la dynamique] de ce champ aveugle, c'est, je crois", écrit Barthes, "ce qui distingue la photo érotique de la photo pornographique. La pornographie représente ordinairement le sexe, elle en fait un objet immobile [un fétiche], encensé comme un dieu qui ne sort pas de sa niche; pour moi, pas de punctum dans l'image pornographique; tout au plus m'amuse-t-elle [et encore : l'ennui vient vite]. La photo érotique, au contraire [c'en est la condition même], ne fait pas du sexe un objet central;

8. P. 87. 
elle peut très bien ne pas le montrer; elle entraîne le spectateur hors de son cadre, et c'est en cela que cette photo, je l'anime et elle m'anime. Le punctum est alors une sorte de hors-champ subtil, comme si l'image lançait le désir au-delà de ce qu'elle donne à voir : pas seulement vers le «reste» de la nudité, pas seulement vers le fantasme d'une pratique, mais vers l'excellence absolue d'un être, âme et corps mêlés. Ce garçon au bras étendu, au sourire rayonnant, bien que sa beauté ne soit nullement académique et qu'il soit à demi sorti de la photo, déporté à l'extrême vers un côté du cadre, incarne une sorte d'érotisme allègre; la photo m'induit à distinguer le désir lourd, celui de la pornographie, du désir bon, celui de l'érotisme; après tout, peut-être est-ce une question de "chance»: le photographe a fixé la main du garçon [Mapplethorpe lui-même, je crois] dans son bon degré d'ouverture, sa densité d'abandon : quelques millimètres de plus ou de moins, et le corps deviné n'eût plus été offert avec bienveillance [le corps pornographique, compact, se montre, il ne se donne pas, en lui aucune générosité] : le Photographe a trouvé le bon moment, le kaïros du désir ${ }^{9}$ ".

Dans ce second fragment, c'est l'absence du corps et la main qui constituent le punctum. Mais la main, parce qu'elle arrive à prendre la bonne position au bon moment. Or, Barthes ne semble pas vraiment l'ignorer, il s'agit d'un autoportrait de Mapplethorpe. C'est dire que l'œil de la caméra ne vise pas Mapplethorpe quand celui-ci cadre et que personne ne vise Mapplethorpe quand ce dernier est photographié ${ }^{10}$. Position de permutation où l'absence change de place mais laisse continuellement la trace de cette absence. L'intérêt que porte Barthes à Mapplethorpe ne peut se comprendre aussi que dans ce qu'il cache et

9. P. 93 et 95.

10. Le catalogue titre bien "Self Portrait 1975". 
ce qu'il cache, ce sont toutes les autres photos que devait bien connaître Barthes, Mapplethorpe ayant exposé à Paris en 78 entre autres, et qui sont loin d'être aussi anodines que les deux qu'il réserve à ses lecteurs. La photographie de Mapplethorpe, souvent d'inspiration homosexuelle, oscille entre l'érotisme et la pornographie. Aux deux photos retenues par Barthes, on pourrait substituer dans les mêmes catégories d'autres photos autrement plus audacieuses montrant des scènes que des publics américains ont trouvé particulièrement offensantes ${ }^{11}$. On pourrait donc se poser la question de savoir quel est l'enjeu réel de La Chambre claire et comment il faut lire les deux discours de l'absence qui s'y tiennent : l'absence de la photo de la mère à la suite du décès de cette dernière et l'absence de ce qui constitue l'œuvre de Mapplethorpe. Nous pourrions même retrouver une condensation de ces deux absences dans la photographie qui ouvre le livre, des rideaux cachant le jour de l'extérieur, un coin de chambre, réduit à un oreiller posé sur un bout de divan, dans des teintes de vert et qui porte le titre de "Polaroïd". II est difficile de ne pas y voir là la citation-image des cinquante premières pages de la Recherche sur le sommeil, le rêve et le souvenir, ces pages qu'analysait Barthes dans un séminaire du Collège de France. II n'est pas difficile d'y supposer l'absence de la mère. II est tout aussi difficile de ne pas noter que la première grande période de photographie de Mapplethorpe s'appelle aussi «Polaroïd». Ce qui s'absente, c'est ce qui ne peut se donner à voir, c'est l'objet du désir qui se détruit

11. Par exemple, à la photo de Philip Glass et Robert Wilson, on pourrait substituer celle de Brian Ridley et Lyle Heeter (voir l'illustration) où les deux personnages costumés de cuir sont enchaînés l'un à l'autre dans une évocation de la relation sado-masochiste comme on pourrait aussi substituer un autre autoportrait de Mapplethorpe où il offre à la caméra son postérieur nu dans lequel est fiché l'extrémité d'un fouet qu'il tient d'une main. 


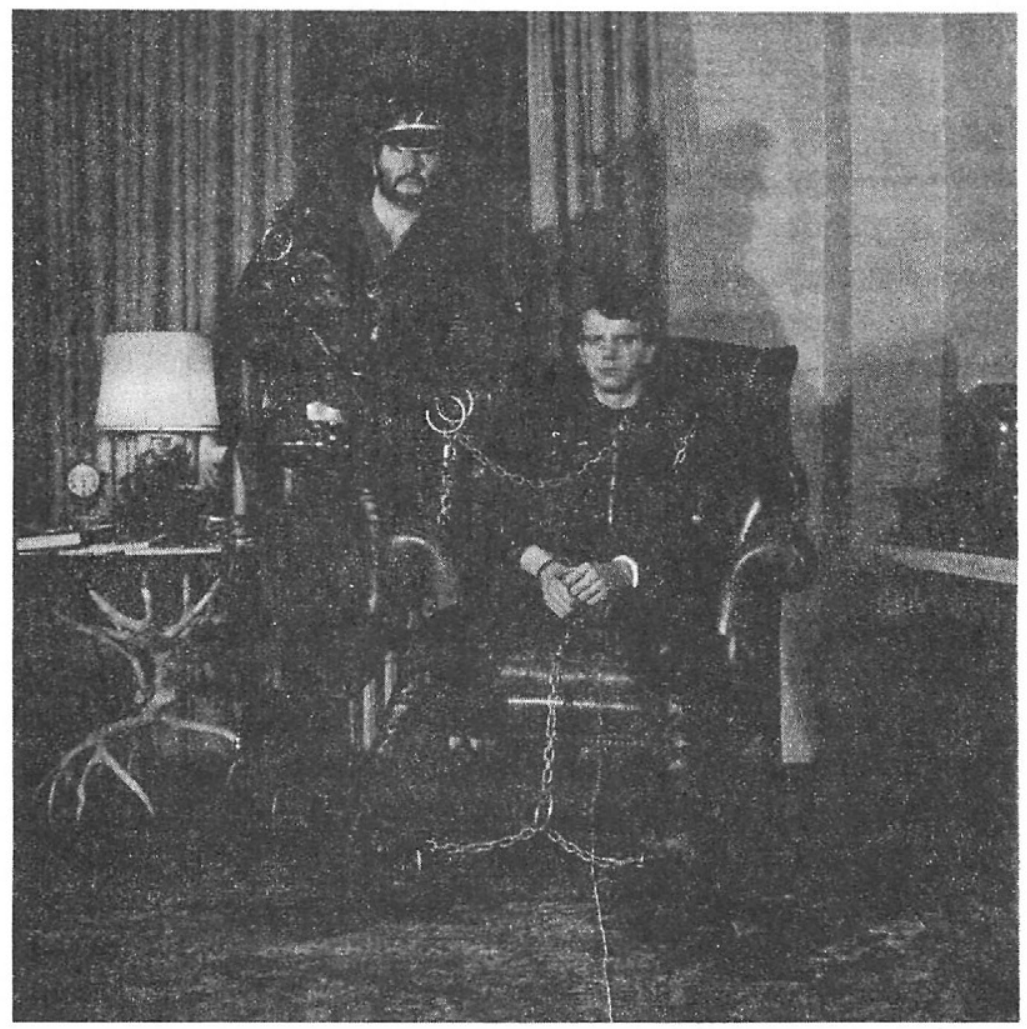

Brian Ridley and Lyle Heeter 1979 
sous le regard. L'érotisme absente la pornographie, le cadrage du sexe.

Ces deux exemples qui auraient pu être encore plus développés permettent de voir comment l'activité sémiotique est conduite. D'une part, les deux seuls objets réels en présence sont les livres. C'est la lecture seule qui permet de transformer ces objets inertes en objets de sens. La lecture s'attache à tel ou tel aspect du discours, de sa présentation, de ce qui est dit et de ce qui n'est pas dit, des rapports que l'on peut poser entre divers éléments... On peut varier le fondement de la lecture, le "ground", au sens peircien. On peut varier son angle, son point de vue, élaborer des interprétances qu'il faut ensuite vérifier en un temps ultérieur. La sémiotique construit ici un objet qui est plus large et plus complexe que les Fragments ou La Chambre claire, un objet qui, en se complexifiant, sollicite non seulement la connaissance de Barthes, mais aussi celle de la photographie et du discours iconique. Le lecteur puise dans ses savoirs de la littérature et file un argument dans le but de convaincre. Mais tout cela n'est possible, sémiotiquement parlant, qu'en créant un objet singulier, un objet dynamique dont la complexité est en expansion. Cet objet se construit, devient provisoirement le sens de la démarche et le plaisir de sa construction, son esthétique. N'importe qui peut reprendre le méme trajet et le poursuivre plus loin ou en inaugurer un autre. C'est en ce sens que l'interprétance comme la semiosis est illimitée sauf à savoir quand il faut, toujours provisoirement sur cette terre, y mettre un point final.

Gilles Thérien, Université du Québec à Montréal 


\section{Bibliographie}

Barthes, Roland, [1975], Roland Barthes, coll. Écrivains de toujours, Paris, Seuil.

Barthes, Roland, [1977], Fragments d'un discours amoureux, Paris, Seuil.

Barthes, Roland, [1980], La Chambre claire, Paris, Cahiers du cinéma, Paris, Seuil.

Marshall, Richard, [1988], Robert Mapplethorpe, Boston, Bulfinch Press.

Peirce, Charles S., [1965-67], Collected Papers, Cambridge Mass., Belknap-Harvard University Press.

Rosset, Clément, [1979], L'objet singulier, Paris, Éditions de Minuit.

Thérien, Gilles, [1990], "Pour une sémiotique de la lecture», Protée, vol. 18, no 2, p. 67-80. 